\title{
El diseño de experimentos en la investigación agrícola
}

The design of experiments in the agriculture research

${ }^{1}$ Carlos Marín Rodriguez a , ${ }^{2}$ Pedro García Mendoza

\section{RESUMEN}

La investigación agrícola posee una gran relevancia para la seguridad agroalimentaria de la población humana actual y futura. Para la año 2050 se prevé que la población mundial alcance cerca de los 10 mil millones de personas y uno de los mayores desafíos será producir alimentos en suficiente cantidad y calidad para alimentar a esta población. Esto no será posible si no se avanza en los procesos de investigación, que permitan generar nuevas tecnologías para la cadena productiva del sector agropecuario. De esta manera, el campo de la estadística aplicada a la producción agrícola se hace cada vez más relevante, por ser la herramienta que le permite a los científicos manejar la información generada en el proceso de investigación, haciéndola racional y amigable, logrando que los resultados puedan ser visualizados con mayor claridad. En este sentido, el objetivo de este trabajo fue presentar una actualización de los conceptos básicos que rigen la investigación científica y los principios fundamentales de la experimentación, con el propósito de generar resultados y conclusiones confiables en la investigación agrícola. El trabajo se basó en la revisión de la bibliografía más reciente y en la experiencia acumulada en los investigadores, autores del tema, haciendo especial hincapié en los aspectos de mayor relevancia y más sensibles para la ciencia agrícola. Se concluye la necesidad que tiene todo científico de tener un razonable entrenamiento estadístico, pero trabando en estrecha colaboración con un estadístico que tenga un amplio conocimiento del campo de aplicación del experimento, lo cual es válido también para otras disciplinas afines con el área agrícola.

Palabras clave: Diseño de experimentos, investigación agrícola, disciplinas de investigación, entrenamiento estadístico, estadística aplicada, estadística.

\begin{abstract}
Agricultural research has great relevance for the agro-food security of the current and future human population. By the year 2050, the world population is expected to reach close to 10 billion people and one of the greatest challenges will be to produce enough food and quality to feed this population. This will not be possible if progress is not made in the research processes that allow generating new technologies for the productive chain of the agricultural sector. In this way, the field of statistics applied to agricultural production becomes increasingly relevant, as it is the tool that allows scientists to manage the information generated in the research process, making it rational and friendly, making the results can be visualized more clearly. In this sense, the objective of this work was to present an update of the basic concepts that govern scientific research and the fundamental principles of experimentation, with the purpose of generating reliable results and conclusions in
\end{abstract}


agricultural research. The work was based on the review of the most recent bibliography and the experience accumulated in the researchers, authors of the subject, with special emphasis on the most relevant and sensitive aspects for agricultural science. It concludes the need that every scientist has to have a reasonable statistical training, but working in close collaboration with a statistician who has a wide knowledge of the field of application of the experiment, which is valid also for other disciplines related to the agricultural area.

keyword: Design of experiments, agriculture research, research disciplines, statistical training, applied statistic, statistic.

${ }^{1}$ Instituto Nacional de Investigaciones Agrícolas (INIA), Maracay, Venezuela.

${ }^{2}$ Universidad Nacional Autónoma de Tayacaja "Daniel Hernández Morillo" (UNAT). Instituto Nacional de Investigaciones Agrícolas (INIA), Portuguesa, Venezuela.

${ }^{a}$ Ing. Agrónomo 


\section{INTRODUCCIÓN}

La investigación agrícola tiene entre sus fundamentos la fase de experimentación, después del planteamiento de la hipótesis y previa al análisis de los resultados, la cual consiste en probar y comprobar la hipótesis de trabajo. Esto a través de una serie de pasos que deben ser ejecutados con precisión y un nivel de confianza alto. En plena era de la IV Revolución industrial o era del conocimiento, los procesos de investigación se han intensificado de tal manera que se han constituido en la fuente fundamental de los nuevos conocimientos y por ende de la nueva tecnología que hoy domina gran parte de los espacios de la función universitaria e incluso de nuestra vida diaria. Es por ello necesario que permanentemente en el ámbito universitario, se deba tener actualizado los conceptos básicos de la investigación científica y los principios fundamentales de la experimentación o diseño experimental, con el propósito de generar resultados y conclusiones confiables.

En la investigación agrícola y su técnica experimental, en general, trata de estudiar una serie de factores, que combinados entre sí se denominan comúnmente "tratamientos" que son aplicados a parcelas de observación o unidad experimental y posteriormente se toman una o varias medidas sobre los individuos u objetos establecidos en la unidad experimental. Si los individuos u objetos dentro de cada unidad experimental son uniformes entre sí, cualquier comparación entre las medidas observadas mostrará diferencias que pueden ser atribuidas a los tratamientos, con un nivel de confianza alto. Los tratamientos, o factores combinados entre sí, pueden ser de diferente naturaleza: Variedades o cultivares de diferentes especies agrícolas (maíz, frejol, sorgo, arroz, etc.), en producción animal las diferentes raciones de alimentos concentrados, dosis de un plaguicida, las fuentes y concentraciones de fertilizantes para ser aplicados al suelo.

Si el investigador logra mediante la realización de un ensayo comparativo, separar las diferencias entre los tratamientos, de la variación debida a múltiples factores no controlados, que agrupamos bajo la común denominación del error experimental, podrá tener una idea muy precisa de la magnitud relativa de las diferencias entre tratamientos al comparar las diferencias entre ellos con la variación no controlada o error experimental. Por lo tanto, el error experimental es una causa de variación y el tamaño, la magnitud o el valor numérico que toma dicha variación en un experimento es su consecuencia o efecto.

Este valor numérico se conoce como desviación típica o error estándar por unidad experimental. Los objetivos del presente trabajo son la de presentar los conceptos actualizados del diseño de experimentos, exponer los diseños experimentales más utilizados en el campo de la investigación agrícola y la de crear una guía 
de consulta en la planeación de ensayos experimentales para proyectos y trabajos de investigación de investigadores, profesores, estudiantes y usuarios especializados.

Para conducir un experimento de forma eficaz y eficiente, sería ideal contar con un experimentador con suficientes conocimientos de los métodos de la Estadística como para planear adecuadamente el ensayo o ensayos. Ello no siempre es posible, debido a que la estadística como ciencia, ha evolucionado tan ampliamente, que constituye una especialidad que debe ser estudiada separadamente para obtener los mejores resultados. En conclusión lo más deseable es que el experimentador, armado de un razonable entrenamiento estadístico, trabaje en estrecha colaboración con un estadístico que tenga un razonable conocimiento del campo de aplicación del experimento.

\section{Conceptos fundamentales del diseño de experimentos}

\section{Diseño experimental}

El diseño experimental es el procedimiento de planeación y conducción de ensayos, así como la definición del análisis estadístico para evaluar los resultados, con el objetivo de tener conclusiones, sobre la hipótesis de trabajo, válidas y objetivas (Montgomery, 2004). El procedimiento incluye la definición de factores a modificar, la manera de su aplicación y el número de pruebas a realizar. También, está determinado por el tipo de aleatorización que se asigna los tratamientos a cada una de las unidades experimentales.

\section{Importancia del diseño experimental}

Proporciona una cantidad máxima de información a la solución del problema en investigación. Y permite ahorrar tiempo y esfuerzo al aplicar el diseño en una sola muestra y no en toda la población que se desea estudiar.

\section{Principios básicos}

Repetición: Significa que el experimento básico o las unidades experimentales se replican dos o más veces, su función es la de suministrar una estimación del error experimental y brindar una valoración más precisa de los efectos de los factores o tratamientos reduciendo el margen de error.

Aleatorización: Es la asignación de tratamientos a unidades experimentales, de modo que todas ellas tengan iguales probabilidades de recibir un tratamiento. $\mathrm{Su}$ función es asegurar estimaciones imparciales de medida de tratamiento y del error experimental.

Control Local: Permite ciertas restricciones sobre la selección aleatoria a fin de reducir el error experimental. El control local hace más extensiva cualquier prueba de significancia.

Rev. Tayacaia 1(2): $64-81,2018$ ISSN:2617-9156 


\section{Variables que intervienen en la respuesta de un fenómeno}

Variable experimental o factor: Es el elemento que tiene como objetivo la generación de resultados. La existencia de un factor de cambio o de acción puede darse espontáneamente o no, voluntaria o involuntariamente, de modo medible o no. El factor puede dividirse en niveles, los cuales pueden ser aplicados de forma aleatoria sobre cada unidad experimental.

\section{Otros conceptos de tratamientos o}

factores: Es el estímulo o modificación que se aplicará a las unidades experimentales, (sustancia, técnica, un proceso), mediante la aplicación de uno o más niveles de un factor o tratamientos a las unidades experimentales se generará una respuesta (Infante, 1997).

Implica el conjunto particular de condiciones experimentales que deben imponerse a una unidad experimental dentro de los confines del diseño seleccionado, tratamiento es cualquier procedimiento, método o estimulo cuyos efectos se desean estimar y comparar. (Rodríguez, 1991).

La variable endógena, no está en estudio pero puede ser controlada, la variable exógena no está en estudio y no es posible controlarlas en forma económica o no se consideran importantes en la manifestación de la respuesta.

Según la escala de medida las variables se dividen en: Variables cuantitativas:
Continuas y discretas. Y Variables cualitativas: Ordinales y nominales.

Variable continua: Puede tomar cualquier valor en un intervalo dado y la escala de medida es infinita.

Variable discreta: Puede tomar sólo un valor en los números naturales y está basada en conteos.

\section{Análisis de la Varianza (ANAVAR):} Proporciona un buen resumen de los cálculos acerca de la variabilidad, resulta de gran utilidad en el estudio de regresiones curvas, así como para hacer comparaciones entre más de dos medios (Snedecor y Cochran, 1979).

Error experimental: Son variaciones extrañas que tienden a encubrir ciertos efectos (Cochran y Cox, 1980).

Son variaciones que se manifiestan en los resultados al aplicar los tratamientos a las unidades experimentales, se clasifican en 2 grupos:

Variaciones pertinentes: variaciones debidas a los efectos de los tratamientos si estos producen efectos distintos. Variaciones no pertinentes: debidas a causas extrañas que disfrazan los efectos del tratamiento (Reyes, 1978).

Describe el fracaso de llegar a resultados idénticos con dos unidades experimentales tratadas idénticamente. (Rodríguez, 1991). Es importante aclarar que el error experimental no es sinónimo de equivocación, sino que es la medida de la variabilidad entre unidades 
experimentales homogéneas a las cuales se les aplicó un mismo tratamiento. Lo anterior en agricultura es muy común, ocasionalmente se trabaja con individuos que provienen de una misma camada, mismo sexo y que son sometidos al mismo tratamiento y encontramos que la respuesta no es idéntica, esto se debe a que cada individuo está sujeto a variables exógenas como el código genético y de esa manera responden diferente a las variables experimentales y endógenas.

\section{Diferencia entre un modelo matemático y un estadístico}

a) El modelo estadístico es un modelo matemático en el cual interviene un elemento aleatorio que denotaremos por medio de $\mathrm{E}_{\mathrm{ij}} \mathrm{y}$ que se refiere al error experimental.

b) El modelo estadístico, en contraste con el modelo matemático, no se aplica a un solo evento, sino a un conjunto de ellos.

c) En el modelo estadístico, a causa de un elemento aleatorio que interviene en él, no se puede determinar con exactitud el valor de una variable en función de otra. En el modelo matemático, si es posible determinar el resultado, por esta razón se le llama modelo determinístico.

Unidad Experimental: La unidad experimental es el elemento (planta, animal u objeto) al que se le modificarán en forma planeada factores para revisar su respuesta.

Testigo o Control: Es la selección de un grupo de unidades experimentales al que no se le aplica ningún nivel de un factor o tratamiento con el fin de comparar los resultados con aquellas unidades que si tienen tratamientos.

Repetición: Las repeticiones serán el número de veces que un tratamiento se aplica al mismo número de unidades experimentales.

Bloque: Es un conjunto de unidades experimentales de los más homogéneo posible que reciben el mismo grupo de tratamientos o factores que se desean comparar.

Suma de cuadrados (SC): Se define como como los efectos cuadráticos de cada una de las fuentes de variación que intervienen en el experimento, en otras palabras se puede resumir como la sumatoria de las diferencias de la media de cada tratamiento con respecto a la media general del ensayo, elevadas al cuadrado.

La suma de cuadrados se puede definir mediante la siguiente ecuación:

$$
S C=\left(Y_{i}-\bar{Y}\right)^{2} \text {; }
$$

De esta ecuación se puede obtener la suma de cuadrados corregida $\left(\mathrm{SC}_{\mathrm{c}}\right)$, la cual es resultado de la diferencia entre la $\mathrm{SC}$ sin corregir $\left(\mathrm{SC}_{\mathrm{sc}}\right)$ y el factor de corrección (FC), tal como se muestra en la siguiente expresión:

$$
S C_{c}=\sum Y^{2}-\frac{\left(\sum Y\right)^{2}}{n}
$$


Grados de libertad: $\mathrm{n}-1$, para el caso de estudios basados en una muestra se ha establecido la restricción de un grado de libertad sobre el tamaño de la muestra.

La varianza $\left(\mathrm{s}^{2}\right)$ se define como el desvío medio cuadrático de las observaciones con respecto a la media, o como el Cuadrado Medio de la fuente de variación objeto de estudio. Se pueden identificar dos elementos, el numerador conformado por la suma de cuadrados corregida y el denominador integrado por los grados de libertad

$$
S^{2}=\frac{\sum Y^{2}-\frac{\left(\sum Y\right)^{2}}{n}}{n-1}
$$

\section{Inferencia estadística}

El análisis estadístico descriptivo de los datos debe realizarse previo a la inferencia, sobre todo para darle consistencia, corregir posibles errores de transcripción o de toma de lecturas de las medidas y verificar supuestos de normalidad. Una vez confirmada la normalidad de la distribución de los datos el proceso de inferencia estadística podrá ejecutarse eficientemente.

La inferencia estadística consiste en el proceso de estimación de los parámetros de la población que dio origen a los datos obtenidos de la muestra en estudio. Estos parámetros son: La media poblacional $\mu$, la varianza $\sigma^{2}, \mathrm{~N}$ tamaño de la población y $\pi$ proporción.

\section{Estimación de medias poblacionales}

En la estimación de las medias de la población que da origen a la media de la muestra, se emplea el Intervalo de confianza, el cual está basado en el error estándar o típico de la media $\left(\mathrm{Se}_{\mathrm{x}}\right)$ y un valor tabulado sobre la distribución de $t$ de Student, para muestras pequeñas $n \leq 30$, o valor normal de $\mathrm{Z}$, para muestras grandes o $\mathrm{n}>30$. Es muy empleado y a su vez recomendado en ensayos experimentales agrícolas fijar un nivel de confianza $\mathrm{p}=0,95$.

El error estándar se puede estimar como:

$$
S e_{\bar{x}}=\frac{s}{\sqrt{n}}
$$

Dónde: S es la desviación estándar y n el número de individuos objeto de la muestra. Entonces para los intervalos de confianza a un $95 \%$ de confianza, se tiene:

a) Estimación de a partir de muestras grandes:

$$
I C_{(0,95)}=S e_{\bar{x}} \times Z_{(\propto<=0,05)} ; n>30
$$

b) Estimación de a partir de muestras pequeñas:

$I C_{(0,95)}=S e_{\bar{x}} \times t_{(n-1 g l ; \alpha=0,05)} ; \mathrm{n} \leq 30$

\section{Contraste de hipótesis}

En el caso de dos muestras $(\mathrm{k}=2)$, que pueden ser relacionadas (dependientes) o no relacionadas (independientes), se emplea con frecuencia la Prueba $\mathrm{t}$ de Student. Dos muestras están relacionadas cuando las medidas u observaciones están 
tomadas sobre el mismo elemento o individuo que forma parte de la muestra, se cumple en casos de medidas tomadas antes y después de un espacio de tiempo.

Por el contrario dos muestras no están relacionadas cuando se tienen un conjunto de elementos o individuos muestreados para un grupo que son diferentes a los elementos o individuos del otro grupo.

Para ambos casos y las pruebas estadísticas en general se emplea un sistema de hipótesis que parte de la igualdad entre los grupos a comparar, hasta determinar si un grupo tiene mayor efecto que otro. Explicado estadísticamente, se comparan las medias entre grupos para determinar si provienen o no de la misma población. Para ello se puede establecer tres sistemas de hipótesis diferentes:

a) Ambos grupos (muestras) son iguales:

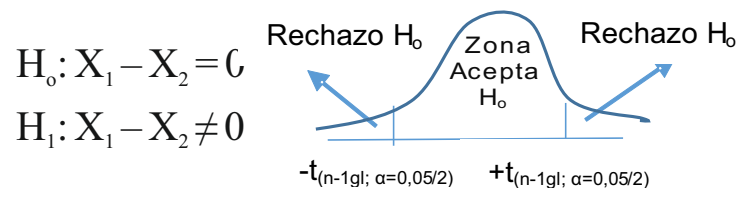

b) El grupo uno es mayor:

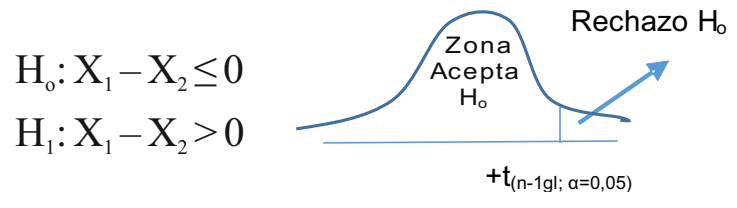

c) El grupo uno es menor:

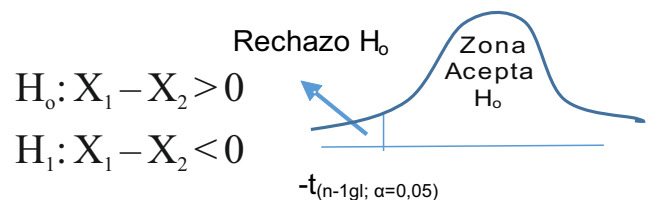

Posteriormente, se calculan los estadísticos de prueba según corresponda el caso de muestra relacionada o no relacionada, los cuales se comparan con los estadísticos tabulados y se decide si se acepta o no la hipótesis nula, o se obtiene el área bajo la curva que corresponda con el estadístico de prueba, es más fácil con el uso de la Hoja electrónica de cálculo o con laguna aplicación estadística como el Infostat, Statistix, Jump, R, Statgraphics, Minitab, SAS University edition, SPSS. (Steel y Torrie, 2008).

\section{Caso k muestras o k-tuples}

Cuando se trata de más de dos muestras en estudio (k-tuples) entra en acción el diseño de experimentos, mediante el cual se disponen los mejores arreglos de niveles de un factor o factores, comúnmente conocidos como tratamientos, repetidos varias veces hasta alcanzar un diseño de campo óptimo, de manera que se puedan tomar las observaciones o medidas con la menor ocurrencia de factores no deseados en el experimento.

De acuerdo al número de niveles de un factor o de combinaciones de niveles de dos o más factores (tratamientos), existen modelos experimentales para $k$ muestras relacionadas y para $k$ muestras no relacionadas. De acuerdo al objetivo de este trabajo se enfocará a la presentación de los diseños experimentales básicos y recomendados en la investigación agrícola bien sea en laboratorio o en campo, los cuales están referidos a $\mathrm{k}$ 
muestras relacionadas.

\section{Supuestos del análisis de varianza}

Homogeneidad de varianza: Las varianzas de las diferentes medias son homogéneas. En el análisis de varianza utilizamos un promedio de $\mathrm{n}$ varianzas para obtener la mejor estimación de la varianza común. Pero si las varianzas dentro de tratamientos fuesen distintas, no tendríamos justificación para combinarlas, ya que el promediar varianzas de tratamientos mayores y menores podría arrojar resultados engañosos. Las pruebas más utilizadas y recomendadas para el estudio de la homocedasticidad de la distribución de los datos muestrales son la de Bartlett y la Q de Cochran.

Normalidad: Las desviaciones del supuesto de normalidad no afectan muy seriamente la validez del análisis de varianza, sin embargo es importante hacer notar que el error experimental se debe distribuir normalmente lo anterior se logra si tomamos en cuenta los principios básicos del diseño experimental, los cuales son repeticiones suficientes del experimento básico, respeto a la aleatorización en la asignación de los tratamientos a las unidades experimentales y control local de las variables endógenas. Existen numerosas pruebas para detectar normalidad en la distribución de los datos, entre la más usadas están los coeficientes de Curtosis y Asimetría y la prueba de Shapriro-Wilk.
Aditividad y linealidad del modelo: Los efectos principales son aditivos, para cada diseño experimental existe un modelo matemático denominado modelo lineal aditivo el cual resume la totalidad de variables endógenas y experimentales que intervendrán en el estudio. La prueba de Tukey tiene el suficiente poder para detectar los efectos lineales y no lineales y es concluyente para determinar aditividad.

Independencia: Las varianzas y las medias de las distintas muestras no están correlacionadas. En algunos datos existe una relación definida entre las medias de las muestras y sus varianzas. Una correlación positiva entre medias y varianzas suele encontrarse cuando existe un amplio rango de medias de la muestra o bien cuando se presenta heterogeneidad de varianza entre los tratamientos considerados en el estudio de algún fenómeno de respuesta. La prueba de las Rachas es potente en la detección de sesgo o conglomerados en los datos muestreados, Rodríguez, 1991.

\section{Diseños experimentales en la Investigación Agrícola}

Existen cuatro diseños experimentales básicos empleados en la investigación agrícola, que son muy utilizados hoy en día y han tenido variantes que han permitido mantenerlos actualizados $\mathrm{y}$ potenciado su uso en casi todos los 
ámbitos de la investigación científica.

Estos son: Diseño totalmente aleatorizado DTA, diseño de bloques completos al azar (DBA), diseño en cuadrado latino (DCL) y diseño en látice (DL), los cuales se presentan a continuación con su modelo lineal aditivo y esperanza de los cuadrados medios.

a) Diseño totalmente aleatorizado, análisis de un factor o una cola o de tratamientos simples.

Es muy utilizado en laboratorio, invernadero y en el campo, depende de que las condiciones ambientales estén muy controladas y la ocurrencia de error sea mínima. En el ejemplo se desarrolla cuando se disponen de cinco niveles de un factor o tratamientos repetidos cuatro veces (Figura 1):

\begin{tabular}{|c|c|c|c|c|}
\hline T1 & T5 & T2 & T4 & T4 \\
\hline T3 & T2 & T4 & T5 & T3 \\
\hline T5 & T3 & T5 & T1 & $\mathrm{T} 2$ \\
\hline T1 & T4 & T3 & T2 & T1 \\
\hline
\end{tabular}

Figura 1. Plano de un DTA con cinco niveles de tratamiento y cuatro repeticiones dispuestos aleatoriamente.

La idea es que cada tratamiento o nivel tenga la oportunidad de confrontarse al menos una vez con el resto de los tratamientos. El modelo lineal aditivo sería:

$$
Y_{i j}=\mu+T_{i}+e_{i j}
$$

Dónde:

$\mathrm{Y}_{\mathrm{ij}}$ : Es la respuesta del tratamiento o nivel del factor estudiado en la unidad experimental correspondiente al i-ésimo tratamiento y a la j-ésima repetición.

$\mu$ : Corresponde a la media general del ensayo o experimento.

$\mathrm{T}_{\mathrm{i}}$ : Es el efecto del i-ésimo tratamiento.

$\mathrm{e}_{\mathrm{ij}}$ : Es el error asociado en la unidad experimental correspondiente al i-ésimo tratamiento y a la j-ésima repetición.

La esperanza de los cuadrados medios o anavar se presenta en la siguiente tabla:

Tabla 1. Esperanza de los cuadrados medios (anavar) en un diseño totalmente aleatorizado partiendo de 5 tratamientos y 4 repeticiones.

\begin{tabular}{lllll}
\hline \multicolumn{1}{c}{$\begin{array}{c}\text { Fuente de } \\
\text { variación }\end{array}$} & $\begin{array}{c}\text { Grados de } \\
\text { libertad }\end{array}$ & $\begin{array}{c}\text { Suma de } \\
\text { cuadrados }\end{array}$ & $\begin{array}{c}\text { Cuadrados } \\
\text { medios }\end{array}$ & Falculado \\
\hline $\begin{array}{l}\text { Entre } \\
\text { Tratamiento }\end{array}$ & $(5-1)=4$ & $\mathrm{SC}_{\text {Trat }}$ & $\mathrm{CM}_{\text {Trat }}$ & $\mathrm{CM}_{\text {Trat }} / \mathrm{CM}_{\text {TratxRep }}$ \\
$\begin{array}{l}\text { Dentro } \\
\text { Tratamiento }\end{array}$ & $5(4-1)=15$ & $\mathrm{SC}_{\text {TratxRep }}$ & $\mathrm{CM}_{\text {TratxRep }}$ & \\
Total & $(5 \mathrm{x} 4)-1=19$ & $\mathrm{SC}_{\text {Total }}$ & $\mathrm{CM}_{\text {Total }}$ & \\
\hline
\end{tabular}

Una vez obtenido el $\mathrm{F}_{\text {calculado }}$ se procede a estimar el área bajo la curva que le corresponde o nivel de probabilidad. Si el nivel de $\mathrm{p}<0,05$ entonces se rechaza la hipótesis nula de lo contrario se acepta que no hay suficiente evidencia en los datos como para detectar diferencias significativas.

b) Diseño de bloques completos al azar (DBA), análisis de dos factores o de dos colas o de tratamientos arreglados dentro de bloques o repetición.

También es utilizado en laboratorio e invernadero y con más frecuencia en el campo, depende de que las condiciones ambientales estén controladas a excepción de la presencia de un gradiente 
de algún factor exógeno que es posible controlar a través de un Bloque, como es el caso de una pendiente de terreno o gradiente de fertilidad o heterogeneidad de las condiciones del suelo. En estos casos, se deben colocar los bloques de tratamientos a lo largo de la pendiente o curva de nivel, o dentro de zonas o paños homogéneos dentro de un suelo heterogéneo. Con ello se lograría en el campo que la ocurrencia de error sea mínima. En el ejemplo se desarrolla cuando se disponen de cinco niveles de un factor o tratamientos repetidos cuatro veces (Figura 2).

La idea es que cada tratamiento o nivel tenga la oportunidad de confrontarse al menos una vez con el resto de los tratamientos. El modelo lineal aditivo sería:

$$
Y_{i j}=\mu+T_{i}+B_{j}+e_{i j}
$$

Dónde:

$\mathrm{Y}_{\mathrm{ij}}$ : Es la respuesta del tratamiento o nivel del factor estudiado en la unidad experimental correspondiente al i-ésimo tratamiento y al j-ésimo bloque o repetición.

$\mu$ : Corresponde a la media general del ensayo o experimento.

$\mathrm{T}_{\mathrm{i}}$ : Es el efecto del i-ésimo tratamiento.

$B_{j}$ : Es el efecto del j-ésimo bloque.

$\mathrm{e}_{\mathrm{ij}}$ : Es el error asociado en la unidad experimental correspondiente al i-ésimo tratamiento y al j-ésimo bloque.

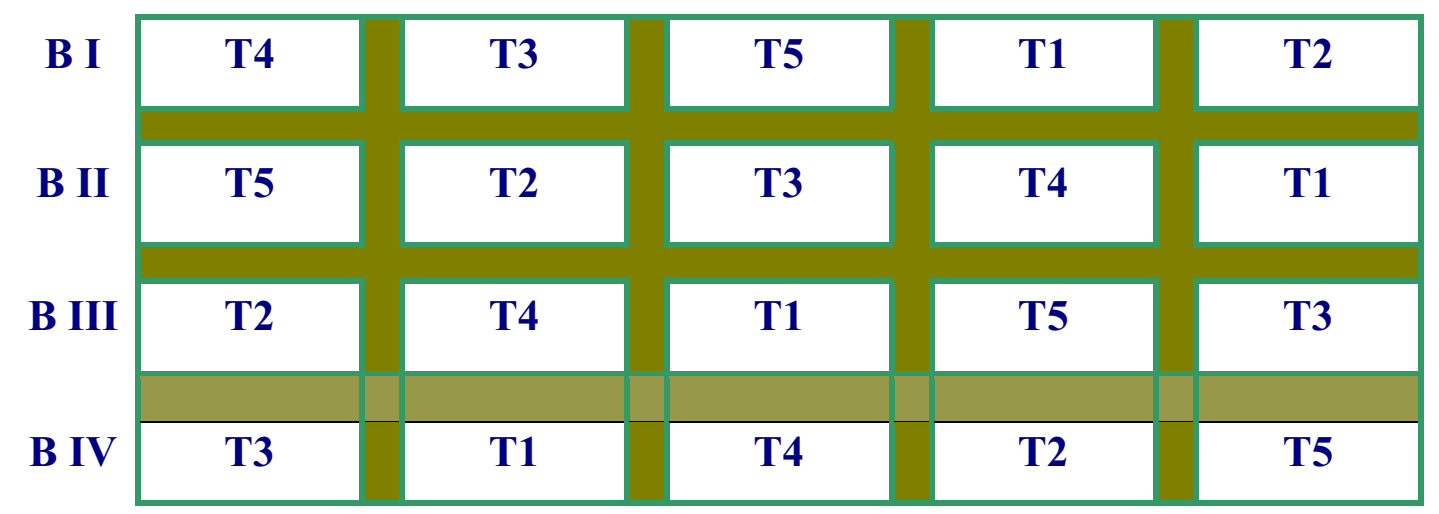

Figura 2. Plano de un DBCA con cinco niveles de tratamiento y cuatro bloques o repeticiones dispuestos aleatoriamente.

La esperanza de los cuadrados medios o anavar se presenta en la tabla 2:

Tabla 2. Esperanza de los cuadrados medios (anavar) en un diseño de bloques completos al azar, partiendo de cinco tratamientos y cuatro bloques.

\begin{tabular}{lllll}
\hline \multicolumn{1}{c}{$\begin{array}{c}\text { Fuente de } \\
\text { variación }\end{array}$} & \multicolumn{1}{c}{$\begin{array}{c}\text { Grados de } \\
\text { libertad }\end{array}$} & $\begin{array}{l}\text { Suma de } \\
\text { cuadrados }\end{array}$ & $\begin{array}{c}\text { Cuadrados } \\
\text { medios }\end{array}$ & F calculado \\
\hline Tratamiento & $(5-1)=4$ & $\mathrm{SC}_{\text {Trat }}$ & $\mathrm{CM}_{\text {Trat }}$ & $\mathrm{CM}_{\text {Trat }} \mathrm{CM}_{\text {Error }}$ \\
Bloque & $(4-1)=3$ & $\mathrm{SC}_{\text {Bloq }}$ & $\mathrm{CM}_{\text {Bloq }}$ & $\mathrm{CM}_{\text {Bloq }} / \mathrm{CM}_{\text {Error }}$ \\
Error & $(4 \times 3)=12$ & $\mathrm{SC}_{\text {Error }}$ & $\mathrm{CM}_{\text {Error }}$ & \\
Total & $(5 \times 4)-1=19$ & $\mathrm{SC}_{\text {Total }}$ & $\mathrm{CM}_{\text {Total }}$ & \\
\hline
\end{tabular}


Una vez obtenido el $\mathrm{F}_{\text {calculado }}$ para tratamiento y para bloque, se procede a estimar el área bajo la curva que le corresponde o nivel de probabilidad de cada una de las F. Si el nivel de $\mathrm{p}<0,05$ entonces se rechaza la hipótesis nula de lo contrario se acepta que no hay suficiente evidencia en los datos como para detectar diferencias significativas tanto para tratamiento como para bloque. En el caso de bloque cuando se rechaza la hipótesis nula con un alto nivel de significación, se dice que el bloqueo fue eficiente porque capturó gran parte de la variación que en ausencia de bloques se le atribuiría al error, lo cual afectaría la potencia del análisis.

a) Diseño en cuadrado latino (CL), análisis de tres factores o de tratamientos arreglados dentro de filas y columnas

También es utilizado en laboratorio e invernadero y con más frecuencia en el campo, depende de que las condiciones ambientales estén controladas a excepción de la presencia de dos gradientes de dos factores exógenos que son posibles de controlar a través de una Fila y una Columna, como es el caso de una pendiente de terreno y gradiente de fertilidad o heterogeneidad de las condiciones del suelo. En estos casos, se deben colocar las filas de tratamientos a lo largo de la pendiente o curva de nivel, y dentro de zonas o paños homogéneos dentro de un suelo heterogéneo arreglados en columnas. Con ello se lograría en el campo que la ocurrencia de error sea mínima. En el ejemplo se desarrolla cuando se disponen de cinco niveles de un factor o tratamientos distribuidos en cinco filas y cinco columnas (Figura 3 ).

La idea es que cada tratamiento o nivel tenga la oportunidad de confrontarse al menos una vez con el resto de los tratamientos. El modelo lineal aditivo sería:

$$
\mathrm{Y}_{\mathrm{ijk}}=\mu+\mathrm{F}_{\mathrm{i}}+\mathrm{C}_{\mathrm{j}}+\mathrm{T}_{\mathrm{k}}+\mathrm{e}_{\mathrm{ijk}} ;
$$

Dónde:

$\mathrm{Y}_{\mathrm{ijk}}$ : Es la respuesta del tratamiento o nivel del factor estudiado en la unidad experimental correspondiente a la i-ésima fila y a la j-ésima columna, donde está ubicado el k-ésimo tratamiento.

$\mu$ : Corresponde a la media general del ensayo o experimento.

$F_{i}$ : Efecto de la i-ésima fila.

$\mathrm{Cj}$ : Efecto de la j-ésima columna.

$\mathrm{T}_{\mathrm{k}}$ : Es el efecto del k-ésimo tratamiento. $\mathrm{e}_{\mathrm{ijk}}$ : Es el error asociado en la unidad experimental correspondiente a la i-ésima fila y la j-ésima columna, donde está ubicado el k-ésimo tratamiento.

$\begin{array}{lllll}\mathrm{C}_{1} & \mathrm{C}_{2} & \mathrm{C}_{3} & \mathrm{C}_{4} & \mathrm{C}_{5}\end{array}$

\begin{tabular}{l|c|c|c|c|c|}
\cline { 2 - 6 } $\mathrm{F}_{1}$ & T1 & T2 & T3 & T4 & T5 \\
\cline { 2 - 6 } $\mathrm{F}_{2}$ & T2 & T3 & T4 & T5 & T1 \\
\cline { 2 - 6 }$F_{3}$ & T3 & T4 & T5 & T1 & T2 \\
\cline { 2 - 6 }$F_{4}$ & T4 & T5 & T1 & T2 & T3 \\
\cline { 2 - 6 }$F_{5}$ & T5 & T1 & T2 & T3 & T4 \\
\cline { 2 - 6 } & & \multicolumn{1}{|c}{}
\end{tabular}

Figura 3. Plano de un CL con cinco niveles de tratamiento distribuidos en cinco filas y cinco columnas, cuadrado $5 \times 5$. 
La esperanza de los cuadrados medios o anavar se presenta en la tabla 3:

Tabla 3. Esperanza de los cuadrados medios (anavar) en un diseño en cuadrado latino 5x5, partiendo de cinco tratamientos distribuidos en cinco columnas y cinco columnas.

\begin{tabular}{lllll}
\hline $\begin{array}{c}\text { Fuente de } \\
\text { variación }\end{array}$ & \multicolumn{1}{c}{$\begin{array}{c}\text { Grados de } \\
\text { libertad }\end{array}$} & $\begin{array}{c}\text { Suma de } \\
\text { cuadrados }\end{array}$ & $\begin{array}{c}\text { Cuadrados } \\
\text { medios }\end{array}$ & F $_{\text {calculado }}$ \\
\hline Tratamiento & $(5-1)=4$ & $\mathrm{SC}_{\text {Trat }}$ & $\mathrm{CM}_{\text {Trat }}$ & $\mathrm{CM}_{\text {Trat }} / \mathrm{CM}_{\text {Error }}$ \\
Filas & $(5-1)=4$ & $\mathrm{SC}_{\text {Fila }}$ & $\mathrm{CM}_{\text {Fila }}$ & $\mathrm{CM}_{\text {Fila }} / \mathrm{CM}_{\text {Error }}$ \\
Columnas & $(5-1)=4$ & $\mathrm{SC}$ Col & $\mathrm{CM}_{\text {Col }}$ & $\mathrm{CM}_{\text {Col }} / \mathrm{CM}_{\text {Error }}$ \\
Error & $(5-1) \mathrm{x}(5-2)=12$ & $\mathrm{SC}_{\text {Error }}$ & $\mathrm{CM}_{\text {Error }}$ & \\
Total & $\left(5^{2}\right)-1=24$ & $\mathrm{SC}_{\text {Total }}$ & $\mathrm{CM}_{\text {Total }}$ & \\
\hline
\end{tabular}

Una vez obtenido el $\mathrm{F}_{\text {calculado }}$ para tratamiento, para fila y columna, se procede a estimar el área bajo la curva que le corresponde o nivel de probabilidad de cada una de las F. Si el nivel de $\mathrm{p}<0,05$ entonces se rechaza la hipótesis nula de lo contrario se acepta que no hay suficiente evidencia en los datos como para detectar diferencias significativas tanto para tratamiento como fila y columna. En el caso de las filas y las columnas cuando se rechaza la hipótesis nula con un alto nivel de significación, se dice que el diseño de cuadrado latino fue eficiente porque capturó gran parte de la variación que en ausencia de éste se le atribuiría al error, lo cual afectaría la potencia del análisis.

\section{a) Diseño en látice simple: Cuadrados o rectangulares (DL)}

También es utilizado en laboratorio e invernadero y con más frecuencia en el campo, soporta mayor cantidad de tratamientos que los diseños anteriores y gana más potencia en detectar diferencias o efectos a medida que aumenta el número de tratamientos. Para ello se recurre al arreglo de tratamientos dentro de una repetición, la cual es subdividida en bloques, en un número tal que sea un divisor exacto del número total de tratamientos, de modo similar el arreglo de tratamientos se replica en otra área adyacente. Con ello se lograría en el campo que la ocurrencia de error sea mínima. En el ejemplo se desarrolla cuando se disponen de 25 niveles de un factor o tratamientos distribuidos en cinco bloques, los cuales se encuentran distribuidos en dos repeticiones (Figura 4).

La idea es que cada tratamiento o nivel tenga la oportunidad de confrontarse al menos una vez con el resto de los tratamientos. El modelo lineal aditivo sería:

$$
\mathrm{Y}_{\mathrm{ijk}}=\mu+\mathrm{R}_{\mathrm{j}}+\mathrm{T}_{\mathrm{i}}+\mathrm{T}_{\mathrm{i} \text { (ajustado) }}+\mathrm{B}_{\mathrm{k}}+\mathrm{e}_{\mathrm{ijk}} ;
$$

Dónde:

$\mathrm{Y}_{\mathrm{ijk}}$ : Es la respuesta del tratamiento o nivel del factor estudiado en la unidad experimental correspondiente al i-ésimo tratamiento en el k-ésimo bloque y a la jésima repetición.

$\mu$ : Corresponde a la media general del ensayo o experimento.

$\mathrm{T}_{\mathrm{i}}$ : Efecto del i-ésimo tratamiento. 
$\mathrm{T}_{\mathrm{i}(\text { ajustado) }}$ : Efecto ajustado de tratamientos.

$\mathrm{Rj}$ : Efecto de la j-ésima repetición.

$\mathrm{B}_{\mathrm{k}}$ : Es el efecto del k-ésimo bloque.

$\mathrm{e}_{\mathrm{ij \textrm {k }}}$ : Es el error asociado en la unidad experimental correspondiente a la i-ésimo tratamiento dentro de laj-ésima repetición y k-ésimo bloque.

Repetición I

\begin{tabular}{l|c|c|c|c|c|}
\cline { 2 - 6 } Bloque 1 & $\mathrm{T} 24$ & $\mathrm{~T} 23$ & $\mathrm{~T} 21$ & $\mathrm{~T} 22$ & $\mathrm{~T} 25$ \\
\cline { 2 - 6 } Bloque 2 & $\mathrm{T} 17$ & $\mathrm{~T} 16$ & $\mathrm{~T} 20$ & $\mathrm{~T} 19$ & $\mathrm{~T} 18$ \\
\cline { 2 - 6 } Bloque 3 & $\mathrm{T} 13$ & $\mathrm{~T} 14$ & $\mathrm{~T} 15$ & $\mathrm{~T} 11$ & $\mathrm{~T} 12$ \\
\cline { 2 - 6 } Bloque 4 & $\mathrm{T} 6$ & $\mathrm{~T} 8$ & $\mathrm{~T} 10$ & $\mathrm{~T} 9$ & $\mathrm{~T} 7$ \\
\cline { 2 - 6 } Bloque 5 & $\mathrm{T} 5$ & $\mathrm{~T} 1$ & $\mathrm{~T} 2$ & $\mathrm{~T} 3$ & $\mathrm{~T} 4$ \\
\cline { 2 - 6 } & & & & &
\end{tabular}

Repetición I

\begin{tabular}{|l|l|l|l|l|}
\hline T21 & T24 & T22 & T25 & T23 \\
\hline T20 & T17 & T16 & T18 & T19 \\
\hline T14 & T15 & T13 & T11 & T12 \\
\hline T9 & T8 & T10 & T6 & T7 \\
\hline T2 & T4 & T3 & T5 & T1 \\
\hline
\end{tabular}

Figura 4. Plano de un DL con 25 niveles de tratamiento distribuidos en cinco bloques en forma aleatoria, localizados en dos repeticiones.

\begin{tabular}{lllll}
\hline $\begin{array}{c}\text { Fuente de } \\
\text { variación }\end{array}$ & $\begin{array}{c}\text { Grados de } \\
\text { libertad }\end{array}$ & $\begin{array}{c}\text { Suma de } \\
\text { cuadrados }\end{array}$ & $\begin{array}{c}\text { Cuadrados } \\
\text { medios }\end{array}$ & \multicolumn{1}{c}{ F calculado } \\
\hline Repetición & $(2-1)=1$ & $\mathrm{SC}_{\text {Rep }}$ & $\mathrm{CM}_{\text {Rep }}$ & $\mathrm{CM}_{\text {Rep }} / \mathrm{CM}_{\text {Error }}$ \\
Tratamiento & $(25-1)=24$ & $\mathrm{SC}_{\text {Trat }}$ & $\mathrm{CM}_{\text {Trat }}$ & $\mathrm{CM}_{\text {Trat }} / \mathrm{CM}_{\text {Error }}$ \\
Trat(ajust) & $(25-1)=24$ & $\mathrm{SC}_{\text {Trat(ajust) }}$ & $\mathrm{CM}_{\text {Trat(ajust) }}$ & $\mathrm{CM}_{\text {Trat(ajust })} / \mathrm{CM}_{\text {Error }}$ \\
Bloque & $(5-1)=4$ & $\mathrm{SC}_{\text {Bloq }}$ & $\mathrm{CM}_{\text {Bloq }}$ & $\mathrm{CM}_{\text {Bloq }} / \mathrm{CM}_{\text {Error }}$ \\
Error & $5 \times(5-1)=20$ & $\mathrm{SC}_{\text {Error }}$ & $\mathrm{CM}_{\text {Error }}$ & \\
Total & $(25 \times 2)-$ & $\mathrm{SC}_{\text {Total }}$ & $\mathrm{CM}_{\text {Total }}$ & \\
& $1=49$ & & & \\
\hline
\end{tabular}

Una vez obtenido el $\mathrm{F}_{\text {calculado }}$ para tratamiento y tratamiento ajustado, para bloque y repetición, se procede a estimar el área bajo la curva que le corresponde o nivel de probabilidad de cada una de las F. Si el nivel de $\mathrm{p}<0,05$ entonces se rechaza la hipótesis nula de lo contrario se acepta que no hay suficiente evidencia en los datos como para detectar diferencias significativas tanto para tratamiento $\mathrm{y}$ tratamiento ajustado. En el caso de las repeticiones y los bloques cuando se rechaza la hipótesis nula con un alto nivel de significación, se dice que el diseño de látice simple fue eficiente porque capturó gran parte de la variación que en ausencia de éste se le atribuiría al error, lo cual afectaría la potencia del análisis.

\section{Arreglos factoriales}

Sobre los diseños básicos presentados anteriormente se pueden establecer arreglos de tratamientos o combinaciones de niveles de dos o más factores, los cuales son muy útiles para estudiar los efectos principales de cada factor y de sus interacciones. 
Estos diseños se utilizan para estudiar los efectos producidos por dos o más factores tratamiento y generalmente sus interacciones. En los ejemplos anteriores se definieron los tratamientos con relación a distintos niveles de un único factor de interés (estructura de tratamientos a una vía de clasificación). Cuando los tratamientos se definen mediante la combinación de los niveles de dos o más factores de interés, se dice que el diseño experimental involucra una estructura factorial de tratamientos.

La estructura factorial de tratamientos puede combinarse con distintos tipos de estructura de parcelas (totalmente aleatorizado, bloques, etc.) para generar diversos diseños experimentales. Es posible postular modelos que consideran estructura factorial de tratamientos ya sea en el marco de un diseño totalmente aleatorizado, un diseño en bloques, un diseño en cuadrado latino, etc. Al especificar el modelo, los parámetros que hacen referencia a los efectos de tratamientos (que surgen de la combinación de dos o más factores) deben descomponerse en un conjunto de parámetros que dan cuenta de cada uno de los factores que intervienen para la definición de un tratamiento.

Por ejemplo, si el tratamiento se define por la combinación de niveles del factor dosis y niveles del factor droga, será necesario especificar en el modelo los efectos dosis y tipo de droga por separado. Se pueden adicionar como términos del modelo todas las interacciones posibles entre los factores que intervienen. En cada caso habrá que juzgar si todas o sólo algunas de las interacciones adicionadas al modelo son requeridas. Si por ejemplo se realiza un ensayo con dos factores en un diseño en bloques completos al azar, no es necesario agregar las interacciones de bloque con cada uno de los dos factores de tratamiento.

En experimentos factoriales completos, se estudian todas las posibles combinaciones de los niveles de los factores (tratamientos) en cada repetición del experimento. Los modelos factoriales aditivos son aquellos en los que los términos que modelan la interacción están ausentes. Los modelos aditivos son usados para estudiar los efectos principales de los factores que intervienen en un proceso en el que se sabe que los factores no interactúan entre sí. El efecto principal de un factor se define como el cambio promedio en la respuesta producida entre cualquier par de niveles del factor considerado.

Para ejemplificar este caso se presenta un experimento factorial $2 \times 2$ (dos factores con dos niveles cada uno) en el que la interacción se supone ausente, el cual se ha dispuesto según un diseño completamente aleatorizado. Los factores se han designado como A y B y sus niveles como $\mathrm{A}_{1}, \mathrm{~A}_{2}$ y $\mathrm{B}_{1}, \mathrm{~B}_{2}$. Como existen 4 tratamientos $\left(\mathrm{A}_{1} \mathrm{~B}_{1}, \mathrm{~A}_{1} \mathrm{~B}_{2}, \mathrm{~A}_{2} \mathrm{~B}_{1}, \mathrm{~A}_{2} \mathrm{~B}_{2}\right) \mathrm{y}$ suponiendo que estos no están repetidos, se tienen cuatro parcelas experimentales. Dado que el diseño es completamente 
aleatorizado la asignación de las parcelas a cada uno de los tratamientos es al azar. Un arreglo posible se presenta en la siguiente Tabla 5:

Tabla 5. Arreglo de dos factores con dos niveles cada uno generando una combinación de cuatro tratamientos.

\begin{tabular}{ccc}
\hline Factor A & Factor B & Tratamiento \\
\hline A1 & B1 & 1 \\
A1 & B2 & 2 \\
A2 & B1 & 3 \\
A2 & B2 & 4 \\
\hline
\end{tabular}

El modelo para este experimento es el siguiente:

$Y_{i j}=\mu+A_{i}+B_{j}+e_{i j} ; \operatorname{con} i=1,2 ; j=1,2$

Donde $Y_{i j}$ representa la respuesta al iésimo nivel del factor $\mathrm{A}$ y j-ésimo nivel de factor $B, \mu$ representa la media general, $A_{i}$ el efecto que produce el i-ésimo nivel del factor $A, B_{j}$ representa el efecto del $j$ ésimo nivel del factor $\mathrm{B}$ y $\mathrm{e}_{\mathrm{ij}}$ es el error aleatorio asociado a la observación ijésima. Los valores $\mathrm{e}_{\mathrm{ij}}$ usualmente se suponen normales, independientes, con esperanza cero y varianza común $\sigma^{2}$. Cuando los experimentos factoriales no tienen repeticiones, el analista debe suponer que los factores no interactúan para poder estimar la varianza del error experimental. Si este supuesto no se cumple entonces el experimento está deficientemente diseñado y las conclusiones del análisis pueden ser completamente erróneas, ya que la interacción será confundida con el error experimental.
Cuando se incluyen las interacciones entonces el esquema de factores presentado en la tabla 5 debe repetirse hasta cinco veces, bajo un DTA, BCA, CL o DL cuadrado. Si se replica sobre un DTA el modelo quedaría de la siguiente manera:

$\mathrm{Y}_{\mathrm{ijk}}=\mu+\mathrm{A}_{\mathrm{i}}+\mathrm{B}_{\mathrm{j}}+\mathrm{AB}_{\mathrm{ij}}+\mathrm{e}_{\mathrm{ijk}} ; \operatorname{con} \mathrm{i}=1,2$; $\mathrm{j}=1,2 \mathrm{y} \mathrm{k}=1,2,3,4,5$

Donde $\mathrm{Y}_{\mathrm{ijk}}$ representa la respuesta al iésimo nivel del factor $\mathrm{A}$ y j-ésimo nivel de factor $\mathrm{B}$ en la k-ésima repetición, $\mu$ representa la media general, $\mathrm{A}_{\mathrm{i}}$ el efecto que produce el i-ésimo nivel del factor $\mathrm{A}$, $B_{j}$ representa el efecto del j-ésimo nivel del factor $\mathrm{B}, \mathrm{AB}_{\mathrm{ij}}$ representa el efecto de la interacción entre los niveles de los factores A y B y $\mathrm{e}_{\mathrm{ij}}$ es el error aleatorio asociado a la observación ij-ésima.

\section{Conclusiones}

Se concluye la necesidad que tiene todo científico de armarse de un razonable entrenamiento estadístico, pero trabajando en estrecha colaboración con 
un estadístico que tenga un amplio conocimiento del campo de aplicación del experimento, lo cual es válido también para otras disciplinas afines con el área agrícola, en fin, lo que significa el trabajo en equipos multidisciplinario.

Además, se generó una fuente de información sobre la teoría del diseño de experimentos y sus aplicaciones en el campo de la investigación científica de tal manera que puede ser consultada como referencia y una guía para investigadores, profesores y estudiantes universitarios en el planeamiento de ensayos experimentales que deben ser incorporados en los trabajos de investigación, en los trabajos de ascenso y las tesis de grado y posgrado que exige el campo universitario y la investigación agrícola.

\section{REFERENCIAS BIOLOGRAFICAS}

Alvarez, L.J., Delrieu, J.C. y Jareño, J. (1993). Tratamiento de predicciones conflictivas: Empleo eficiente de información extramuestral. Estadística Española, 35: 439-461.

Cochran, W.G. y Cox, G.M. (1985). Diseño de experimentos. Editorial Trillas México. 661 p.

Collings, B.J.; Hamilton, M.A. (1988). Estimating the power of the twosample Wilcoxon test for location shift. Biometrics, 44: 847-860.

Di Rienzo, J; Casanoves, F.; Gonzalez, L.; Tablada, E.; Díaz M.; Robledo, C.;
Balzarini, M. (2001). Estadística para las Ciencias Agropecuarias. 4 ta Ed. Triunfar. Córdoba, Argentina.

Efron, B; Tibshirani, R. (1993). Bootstrap methods for standard errors, confidence intervals, and other methods of statistical accuracy. Statistical Science, 1(1): 54-77.

Graybill, F.A. (1961). An Introduction to Linear Statistical Models. McGraw Hill, New York.

Hinkelmann, K.; Kempthorne, O. (1994). Design and Analysis of Experiments. Volume 1: Introduction to Experimental Design. John Wiley \& Sons, Inc., New York.

Hocking, R.R. (1996). Methods and Applications of Linear Models. Regression and the Analysis of Variance. John Wiley \& Sons, Inc., New York.

Huynh, H.; Feldt, L.S. (1976). Estimation of the Box Correction for Degrees of Freedom from Sample Data in the Randomized Block and Split Plot Designs. Journal of Educational Statistics, 1: 69-82.

Jarque, C.M; Bera, A.K. (1987). A test for normality of observations and regression residuals. International Statistical Review, 55: 163-172.

Mahibbur, R.M.; Govindarajulu, Z. (1997). A modification of the test of Shapiro and Wilks for normality. Journal of Applied Statistics, 24(2): 219-235. 
Searle, S.R. (1987). Linear Models for Unbalanced Data. New York, John Wiley \& Sons, Inc., New York.

Shapiro, S.S.; Francia, R.S. (1972). An approximate analysis of variance test for normality. Journal of the American Statistical Association, 67:215-216.

Snedecor, G.W. (1956). Métodos Estadísticos Aplicados a la Investigación Agrícola y Biológica. Compañía Editorial Continental, S.A., México.

Snedecor, G.W.; Cochran, W.G. (1967). Statistical Methods. Ames, IA: Iowa State University Press.

Steel, R.G and Torrie, J.H. (1985). Bioestadística: Principios y procedimientos. 2da Ed., McGraw-Hill Editorial Latinoamericana, Bogotá. 620 p.

Winer, B.J. (1971). Statistical Principles in Experimental Design. 2nd Ed., McGraw-Hill Book Co., New York.

\section{CORRESPONDENCIA}

Msc. Carlos Marín,

e-mail: cmarin@inia.gob.ve

Inv. PI-8. Biometría aplicada a la Investigación Agrícola INIA CENIAP. 